\title{
Lexis
}

Journal in English Lexicology

17 | 2021

Humor, creativity and lexical creation

\section{Humour, création lexicale et créativité linguistique comme stratégies de caractérisation des personnages de fiction : le cas de Barney Stinson dans How I Met Your Mother}

Lucile Bordet

\section{OpenEdition}

\section{Journals}

Édition électronique

URL : https://journals.openedition.org/lexis/5303

DOI : $10.4000 /$ lexis. 5303

ISSN : 1951-6215

Éditeur

Université Jean Moulin - Lyon 3

Référence électronique

Lucile Bordet, « Humour, création lexicale et créativité linguistique comme stratégies de caractérisation des personnages de fiction : le cas de Barney Stinson dans How I Met Your Mother ». Lexis [En ligne], 17 | 2021, mis en ligne le 15 août 2021, consulté le 20 août 2021. URL : http:// journals.openedition.org/lexis/5303; DOI : https://doi.org/10.4000/lexis.5303

Ce document a été généré automatiquement le 20 août 2021.

\section{(†) $\odot$

Lexis is licensed under a Creative Commons Attribution-NonCommercial-NoDerivatives 4.0 International License. 


\title{
Humour, création lexicale et créativité linguistique comme stratégies de caractérisation des personnages de fiction : le cas de Barney Stinson dans How I Met Your Mother
}

\author{
Lucile Bordet
}

\section{Introduction}

1 L'humour, le rire, caractérisent les séries télévisées appartenant au genre des sitcoms (situation comedies). Ces fictions ont fréquemment recours à divers procédés véhiculant l'humour afin de caractériser les personnages. C'est notamment le cas du personnage de Barney Stinson dans la série télévisée américaine How I Met Your Mother, créée par Carter Bays et Craig Thomas et diffusée de 2005 à 2014 aux Etats-Unis. Connu des amateurs de la série pour ses nombreux jeux de mots et ses expressions idiolectales récurrentes, le personnage de Barney incarne dès le premier épisode celui qui fait rire et qui dérange par ses jeux de mots défiant souvent le politiquement correct.

2 Le recours récurrent à des formules idiolectales qui se déclinent tout au long de la série attribue au personnage de Barney Stinson des particularités langagières humoristiques si identifiables qu'elles en deviennent définitoires du personnage mais aussi imitables, tant par les autres personnages que par les amateurs du programme télévisé. Ces éléments humoristiques s'appuient sur des procédés de création lexicale variés (métaphore, troncation, siglaison, amalgame, etc. selon la typologie définie par Tournier [2004]) mais également sur l'homonymie, les détournements phonologiques 
ou encore la syllepse oratoire, faisant alors appel à la création lexicale et/ou la créativité dans un but humoristique.

Cet article se propose d'étudier les diverses stratégies linguistiques qui ont permis de créer de l'humour. Nous nous demanderons comment les auteurs de la série sont parvenus dès le premier épisode, et pendant 9 saisons, à caractériser le personnage de Barney Stinson en jalonnant son discours d'expressions idiolectales, récurrentes mais variées, fondées sur des procédés de création lexicale et en ayant recours à la créativité linguistique. Cela nous conduira à nous interroger sur les points suivants : parmi tous les procédés utilisés, quels sont les plus fréquents? Y a-t-il des procédés de création lexicale plus créatifs et productifs que d'autres et plus aptes à véhiculer l'humour? Si oui, correspondent-ils aux procédés les plus fréquemment utilisés dans le corpus à l'étude? Le recours à des procédés linguistiques particuliers est-il suffisant pour caractériser un personnage de fiction et véhiculer l'humour?

Pour mener à bien cette étude, nous expliciterons dans un premier temps les termesclés et nous exposerons les acceptions retenues pour "création lexicale" et "créativité ». La notion d'" humour » sera également abordée lors d'un rappel des théories de l'humour les plus fréquentes afin de présenter et de justifier le positionnement théorique retenu pour cet article. Nous présenterons ensuite le corpus et expliquerons la méthodologie adoptée pour son traitement. Une analyse du corpus sera ensuite menée en prêtant une attention particulière à l'articulation des diverses occurrences de création / créativité lexicale et de l'humour. L'analyse ne se voudra pas exclusivement morphologique afin, d'une part, d'éviter de proposer un simple catalogue des procédés relevés et, d'autre part, afin de souligner que les matrices lexicogéniques en elles-mêmes ne peuvent suffire à caractériser un personnage et à produire de l'humour. Aussi les thématiques récurrentes dans le discours du personnage de Barney Stinson seront-elles abordées afin de mieux cerner comment s'effectue la caractérisation de ce personnage de fiction.

\section{Définition des termes-clés}

5 La « création lexicale » et la " créativité » sont deux notions qui diffèrent tout en étant complémentaires dans le cadre de l'expression de l'humour. La notion de «création lexicale» sera prise au sens large et inclura donc les cas de néologie morphologique, mais aussi de néologie sémantique. Nous adopterons la typologie des matrices lexicogéniques définie par Tournier [2004]. Si les procédés de création lexicale s'accompagnent en général de créativité, nous considérons que la créativité linguistique n'est pas nécessairement source de création lexicale morphologique ou sémantique en ce que l'expression de l'humour peut se faire par le recours à des lexies existantes dans le cadre de jeux de mots par exemple: If memory serves me, she had a huge 'but'. Her huge butt. Nailed it! (S3E4). Nous postulons également que le recours à des procédés de création lexicale et à la créativité linguistique ne peut être suffisant pour véhiculer l'humour et attribuer des traits de personnalité à un personnage. Comme nous le verrons en 4., c'est une synergie multifactorielle qui semble permettre d'aboutir à ce résultat.

6 Il est également nécessaire de définir ce que nous entendons par "expression de l'humour ", terme que nous emploierons au sens large et qui peut se décliner en 
sarcasme, ironie, etc. lorsque les conditions de création lexicale et de créativité telles qu'elles ont été définies sont réunies.

7 Il ne s'agit pas ici d'ouvrir un débat sur les différentes théories de l'humour ou de tenter de démontrer laquelle serait supérieure aux autres. Il semble toutefois important d'adopter un positionnement théorique et de justifier celui-ci. Pour ce faire, rappelons qu'il existe plusieurs théories de l'humour comme le synthétisent Vandeuren \& Vandeuren [2016]:

- la théorie du sentiment de supériorité et de dégradation du risible (aussi connue sous le nom de théorie morale ou pessimiste) selon laquelle celui qui rit se sentirait supérieur à l'objet de son rire perçu comme dégradé et en devient risible. Le sujet rieur se pense alors supérieur à tout ce qui présente des infériorités, des insuffisances, des imperfections physiques, morales, intellectuelles ou sociales.

- la théorie sociale de Bergson $[1900$; 2000] s'intéresse aux mécanismes du rire. Est risible ce qui ne rentre pas dans la norme ou ce qui relève de la «mécanique plaquée sur du vivant » pour reprendre sa formule (Bergson [1900]). Il affirme aussi que le rire possède un rôle de correcteur social en sanctionnant ce qui dévie des normes sociales. Cette théorie partage des traits communs avec la théorie du sentiment de supériorité et de dégradation du risible en ce que le rieur se considère en position de supériorité et se place en correcteur de la déviance.

- la théorie psychophysiologique ou de la décharge, qui consiste à envisager le rire comme une échappatoire face à une situation de stress ou de tension et donc comme un moyen de relâcher la pression émotionnelle.

- les théories du contraste et de l'incongruité selon lesquelles une situation présentant une incongruité, une ambiguité ou un décalage par rapport à une norme ou un attendu devient source de rire et d'humour, comme l'explique Sørensen [2016: 7] s'appuyant sur Suls [1972] : Humour is a special way of communicating which is based on ambiguity and incongruity. [...] Today, incongruity dominates within humour studies. It is a theory which focuses on the cognitive aspect of how we recognise and mentally process something to be funny. That it is cognitive simply means that it concerns our capacity to understand and perceive the world. In incongruity theory, humour is found to include a discrepancy or ambiguity which forces us to think in more than one dimension at the same time.

- la théorie de la violation bénigne, développée par Veatch [1998], selon laquelle une situation humoristique se produirait en présence de quelque chose perçu comme « un peu incongru, choquant ou déplacé, mais pas trop ». On perçoit ici un lien avec la théorie du contraste et de l'incongruité, mais c'est l'élément choquant ou déplacé, absent de la théorie précédente, qui en fait la spécificité.

8 Notons toutefois que ces théories présentent des limites et ne sauraient rendre compte individuellement et à elles seules de tout ce qui est humoristique. La théorie du sentiment de supériorité et de dégradation du risible, la théorie sociale de Bergson ainsi que la théorie psychophysiologique ou de la décharge ne nous semblent pas pertinentes pour l'analyse du corpus que nous avons constitué à partir d'une sitcom au vu des différentes occurrences relevées. En revanche, une combinaison de la théorie du contraste et de l'incongruité et de la théorie de la violation bénigne semble adaptée pour rendre compte des divers procédés utilisés dans la caractérisation du personnage de Barney. Ses créations lexicales jouent avec la plasticité de la langue menant souvent à des incongruités sur les plans morphologique, syntaxique ou encore thématique. Sur le plan morphologique, on pense notamment au recours au procédé de l'amalgame qui 
produit des occasionalismes composés de fragments de lexies existantes: You're about to be sworn to secrecy to the sacred vows of a bro oath or broath. (S7E19)1. Les incongruités syntaxiques se manifestent dans le jeu sur la grammaticalité ou l'agrammaticalité des structures : He's the awesomest most best lookingest greatest guy ever (S2E10). D'un point de vue thématique, on note le recours fréquent aux domaines tabous (Before she hops on your disco stick (S5E16)), ancrant par là même ces procédés dans la théorie de la violation bénigne et celle de l'incongruité que nous considérons comme complémentaires.

Dans le cadre de cette étude, nous considérons donc comme humoristique toute forme de création lexicale ou de créativité linguistique présentant une incongruité, une ambiguïté, un décalage par rapport à une norme ou un attendu aux niveaux morphologique, syntaxique, sémantique, phonologique, mais aussi thématique.

La perception de l'humour étant primordiale, dans la mesure où quelque chose ne peut faire rire que parce qu'il est perçu comme humoristique dans un contexte qui s'y prête, nous adopterons également la position de McGhee [1979], s'appuyant sur Shaffer \& Kipp [1912:267] qui estiment qu'est humoristique ce qui relève de la capacité à " perceive incongruity, noticing (or creating) a discrepancy between what is usual or expected and what is experienced». La réussite du fait humoristique dans une sitcom tient autant à la construction du discours qu'à sa perception par le téléspectateur.

\section{Corpus et méthodologie de collecte et d'analyse des données}

11 En guise de corpus, cette étude prend appui sur l'ensemble des 9 saisons de la série télévisée américaine How I Met Your Mother. La collecte des données a été effectuée manuellement selon plusieurs critères. Le personnage de Barney étant destiné à faire rire, la quasi-totalité de ses répliques s'accompagne de "rires en boîte ", moyen pour les producteurs de la série télévisée de souligner la dimension humoristique de son discours. Certaines théories évolutionnistes du rire avancent l'hypothèse de la contagion émotionnelle du rire Duchenne ${ }^{2}$. Ce type de rire répondant à des stimuli cognitifs extérieurs est déclenché par les neurones miroirs qui s'activeraient lorsque l'on entend quelqu'un rire et qui provoqueraient la réponse "contagieuse " (voir Hurley, Dennett \& Adams [2013]). Ceci semble expliquer la présence de ces « rires en boîtes » dans le genre télévisuel des sitcoms. Leur finalité étant de faire rire, cet ajout de «rires en boîte» vient renforcer inconsciemment la réponse positive des téléspectateurs au programme télévisé.

12 Toutefois, dans le cadre de cet article, nous avons fait le choix d'écarter certaines formes d'humour ne répondant pas aux principes de création lexicale et/ou de créativité. Nous pensons notamment aux formules liées au comique de répétition, à l'ironie, au sarcasme, ou encore aux diverses blagues ou histoires absurdes narrées par le personnage qui sont pourtant des vecteurs d'humour en raison du décalage, de l'incongruité qu'elles créent ou des tabous qu'elles brisent et des normes qu'elles enfreignent ${ }^{3}$.

En revanche, nous avons fait le choix de conserver toute forme d'humour s'appuyant sur les matrices lexicogéniques définies par Tournier [2004], faisant ainsi preuve de stratégies de création lexicale, mais également de créativité linguistique. Comme on le 
verra en 3., les nombreuses créations lexicales du corpus s'apparentent à des occasionnalismes, à des expressions idiolectales qui sont à contraster avec les néologismes.

En effet, selon Bauer [2004 : 77], un néologisme se définit comme « a newly coined word [that] enters the general vocabulary of the language ». Or, ce n'est jamais le cas des créations lexicales du corpus. Au contraire, elles correspondent davantage à des créations ad hoc dont Bauer [1983:45] dit qu'il s'agit de créations qui répondent à un besoin ponctuel : "new complex word coined by a speaker/writer on the spur of the moment to cover some immediate need". Ces créations lexicales peuvent également s'apparenter à la notion d'« occasionalisme », terme créé par Chanpira [1966]. Selon Dressler \& Tumfart [2017 : 155-156], un occasionalisme correspond à :

a new word created for a poetic function at a specific place in a literary text, and

which has little chance to be accepted by the language community as a neologism.

Si la fonction poétique et la dimension littéraire ne s'appliquent pas au corpus à l'étude, on peut toutefois retenir la seconde partie de la définition qui stipule que ces créations lexicales ont peu de chances d'entrer un jour dans la langue. En ce qui concerne notre corpus, ces créations ad hoc ou occasionnalismes répondent bien à un besoin ponctuel et immédiat ${ }^{4}$ en lien direct avec le genre télévisuel de la série : celui de véhiculer l'humour et dans le même temps de permettre la caractérisation du personnage.

Outre les procédés appartenant aux diverses matrices lexicogéniques, ont également été inclus les nombreux cas reposant sur des jeux phonologiques, l'homonymie, la polysémie et notamment les syllepses oratoires relevant davantage de la créativité que de procédés de création lexicale en ce que les auteurs jouent en discours sur la double interprétation possible d'une même lexie qui possède virtuellement deux acceptions différentes ou plus en langue :

(1) Barney: First we got to pick someone up at the airport. [...]

Ted: Why do you have those suitcases, and who are we picking up?

Barney: I don't know. Maybe her? Or her.

Ted: Wait so when you said you were going to "pick someone up at the airport", you meant you were going to "pick someone up at the airport". (S1E3)

On constate en (1) un jeu sur la polysémie de pick someone up (aller chercher quelqu'un / draguer) et le recours à cette stratégie est relativement fréquent comme on le verra par la suite.

\section{Analyse des données du corpus}

Le corpus présente 354 occurrences relevant de procédés de création lexicale et/ou de créativité linguistique qui ont été classés selon leur type. Soulignons la multiappartenance de certaines occurrences à plusieurs procédés comme en témoigne l'exemple suivant :

(2) Marshall: It's as if a cockroach and a mouse, you know...

Barney: Did the horizontal ten-legged interspecies chacha? (S1E7)

On relève notamment une métaphore ainsi qu'un phénomène de composition dans cet extrait. Dans ces cas, les occurrences ont été comptabilisées dans les différentes catégories de procédés concernés. 
17 Dans un premier temps, les données recueillies dans le corpus ont été réparties selon les différentes matrices lexicogéniques. Afin de ne pas établir un simple catalogue des différents procédés relevés, nous tâcherons premièrement de montrer en quoi chacun des procédés de création lexicale ou procédés créatifs contribue à la construction de l'humour en lien avec les différentes théories de l'humour retenues dans le cadre de cet article, et ce, dans le but de comprendre les mécanismes de caractérisation d'un personnage de fiction humoristique.

Après une présentation des diverses matrices lexicogéniques du corpus ainsi que des stratégies de créativité linguistique, illustrées d'exemples, nous nous concentrerons sur quelques structures idiolectales mais aussi sur les thématiques récurrentes dans le discours de Barney Stinson, et ce, toujours dans l'optique de comprendre les mécanismes linguistiques qui sous-tendent l'expression de l'humour et permettent d'aboutir à la caractérisation d'un personnage de fiction.

\subsection{Matrices lexicogéniques et stratégies de créativité linguistique}

Nous proposons de débuter cette étude par un tour d'horizon des divers procédés de création lexicale mis en œuvre pour véhiculer l'humour dans le discours du personnage de Barney Stinson. Dans cette section, nous proposons une typologie des diverses stratégies de création et/ou de créativité lexicale. L'analyse du corpus a révélé la présence de la plupart des matrices lexicogéniques définies par Tournier [2004], mais en proportions différentes. Il s'agira ensuite de déterminer si certaines matrices sont plus créatives que d'autres et si elles correspondent aux procédés les plus fréquemment utilisés dans le corpus. Le discours de Barney Stinson est émaillé de procédés de création lexicale présentés ci-dessous par ordre de fréquence absolue et illustrés d'exemples.

\subsubsection{La métaphore}

La métaphore est de loin le procédé de création lexicale sémantique le plus représenté dans le corpus avec un total de 77 occurrences ${ }^{5}$, dont 38 s'accompagnent de syllepses oratoires comme en témoignent les exemples ci-après :

(3) Barney: Join the Barnacle in the pharma girl free for all. Side effects may include: loss of clothing, rug burn, shortness of breath and sore abdominals the next morning. (S5E16)

(4) Barney: That the captain moored his dingy in Lily's lagoon? Most definitely. (S9E20)

(5) Barney: What does a fella have to do to get leid around here?

Hula girl: Yeah, right, cos I'm wearing a lei.

Barney: It isn't funny if you explain the joke (S1E6)

(6) Hula girl: You certainly are a charming devil.

Barney: I'm also a horny devil. (S1E6)

(7) Barney: You invited me to your apartment to play battleship! Is that not an internationally recognized term for sex? (S1E14)

(8) Barney: It appears to be some sort of Asian looker. Yes, because on Not a Father's day, you get a thai you'd actually wear. Word play five! (S4E7)

(9) Barney: There's a citizenship test tomorrow. You have to do it. I'll help you study. We're gonna stay up all night long. I'm gonna drill you and then we're gonna study. No, seriously, we're gonna do some cramming and then we're gonna study. No, seriously, we're gonna bone up on... 


\author{
Robin: Ok Barney... \\ Barney: Sorry, it's a rich area. (S5E5) \\ (10) Guys, I've got a new bro. A bro that puts all other bros to shame. The \\ bitches love him. He buries bones all day. No one chases tail like him. Why \\ aren't you guys laughing? Oh, I probably should have led with this: he's a \\ dog. I've named him Brover. I'll go fetch him. (S8E5)
}

En (3), outre le nom "barnacle" utilisé pour renvoyer au personnage de Barney ${ }^{6}$, on note une métaphore médicale filée. C'est également le cas des extraits (9) et (10) qui présentent en sus de la métaphore filée, des cas de syllepses oratoires. Ces dernières sont également présentes dans les occurrences (5) à (10). En (5) et (8), on note en outre un jeu sur la graphie / phonie de lei / lay et thai / tie. En (4) l'adjectif horny s'apparente également à une syllepse que le spectateur interprète comme telle à cause de la dimension visuelle puisque cet épisode se déroule lors d'une soirée costumée pour Halloween et que le personnage de Barney est déguisé en diable et porte une paire de cornes sur la tête. Si la plupart de ces métaphores sont aisément compréhensibles puisqu'elles s'appuient sur des domaines sources et cibles fréquemment associés, l'occurrence (7) ne peut s'interpréter comme une métaphore sexuelle qu'à l'aide du cotexte droit lorsque Barney explique sa méprise et associe sexe et bataille navale (play battleship. Is that not an internationally recognized term for sex?). Parmi les exemples cités, un schéma semble déjà se dessiner quant aux thématiques abordées par ces métaphores, à savoir celles du sexe. Nous y reviendrons en 4.5. lors de l'étude des thématiques les plus fréquentes du corpus. Toutes ces occurrences se révèlent humoristiques en raison de l'incongruité de l'image mentale créée par ces métaphores mais également en raison de la thématique taboue abordée, considérée comme déplacée, qui relève de la théorie de la violation bénigne.

\title{
3.1.2. La composition ou juxtaposition
}

21 La composition ou juxtaposition constitue le second procédé le plus représenté du corpus avec 70 occurrences :

(11) Barney: I'm suit sitting for someone. (S5E13)

(12) Barney: I'm birthday suiting up. (S1E14)

(13) Barney: Now, ladies, slut up! (S1E19)

(14) Barney: Oh yeah, we do that behind your back. Ted out: to overthink.

Also see Ted up: to overthink something with disastrous results. Simple

sentence: Billy tedded up. (S1E7)

(15) Barney: You are legen - daddy, legendaddy. (S6E19)

Les occurrences (11) à (14) contiennent des verbes à particules composés ou phrasal verbs. Les exemples (11), (12) et (13) présentent des cas de composition verbale prenant appui sur des structures composées préexistantes du type babysit ou plus récemment catsit pour (11) et suit up auquel Barney a fréquemment recours, pour (12) et (13). Comme on le verra par la suite en 4 ., lorsque l'on étudiera quelques-unes des stratégies récurrentes de création lexicale, l'une des particularités du discours humoristique de Barney consiste à détourner ou défiger des structures existantes telles que suit up. L'effet humoristique est alors assuré par le décalage entre l'attendu morphosyntaxique du téléspectateur qui a connaissance de la structure existante et l'occasionnalisme créé par le personnage, en vertu des principes de la théorie du contraste et de l'incongruité. L'occurrence (14) qui comporte des phrasal verbs nécessite une explicitation du sens nouvellement créé. En effet, les phrasal verbs sont des constructions complexes dont le 
sémantisme ne peut correspondre à la somme du sens des éléments qui les composent, a fortiori puisque qu'il s'agit d'une création ad hoc impliquant, en plus du phénomène de composition, un processus de conversion du nom propre Ted en verbe. Pour l'exemple (15), l'analyse semble plus complexe : on note d'abord un phénomène de troncation de legendary pour en conserver le segment initial associé à la lexie daddy. Cet occasionnalisme se situe entre la composition et l'amalgame sans entrer pleinement dans l'une ou l'autre des catégories si l'on s'en tient aux définitions traditionnelles de ces procédés.

\subsubsection{L'affixation et plus spécifiquement l'infixation}

Le personnage de Barney a également fréquemment recours au procédé de troncation de lexies en y insérant la locution wait for it dont on peut dire qu'elle joue le rôle d'une incise métadiscursive ${ }^{7}$. Sous l'effet de la répétition du procédé, cette incise métadiscursive peut être considérée comme un infixe discursif et peut à ce titre relever de l'affixation après une étape de troncation. Le corpus ne comprend pas moins de 28 occurrences de ce procédé :

(16) Barney: De - wait for it - nied, denied! (S1E1)

(17) Barney: It's gonna be legen - wait for it and I hope you're not lactose intolerant cause the second part of that word is - dary - legendary! (S1E3)

(18) Barney: Hey, good news. We're all set for the release.

Robin: The what?

Barney: When we leave the church, they're gonna release a 100 doves. It's gonna be avi - wait for it - ary, aviary. (S9E7)

(19) Barney: I'll teach that kid how to be awesome in ways that you and Lily never could. It's gonna be legen - wait for it - no, I won't wait for it and neither should little baby Marvin so maybe it's better if you just die right now - dary. (S8E2)

Comme on peut le constater en (17) et en (19), l'infixe discursif ne se limite pas toujours à la locution wait for it et peut s'accompagner de propositions complètes. L'humour réside ici dans la déconstruction de l'adjectif et l'insertion d'un infixe discursif inattendu, du moins dans les premiers épisodes, qui constitue l'incongruité nécessaire à la réalisation de l'humour. Au fil de l'avancement de la série, cette stratégie est reprise au point de devenir caractéristique du personnage et perd de sa dimension inattendue. Cependant l'humour reste présent, vraisemblablement à cause de l'effet de comique de répétition, mais également parce que la théorie de la violation bénigne s'applique toujours au regard de la construction morphologique.

\subsubsection{La troncation}

Le procédé de troncation est présent à 27 reprises dans le corpus :

(20) Barney: You're welcs. (S7E22)

(21) Barney: You are legen - daddy, legendaddy. (S6E19)

(22) Barney: It's gonna be legen-jerry. (S6E21)

En (20), il s'agit de la troncation de welcome alors qu'en (21) et (22) il y a troncation de legendary. Les deux premières syllabes seulement ont été conservées pour être associées, par le biais d'un amalgame ou d'une composition, selon la position adoptée, aux noms daddy et Jerry à cause de leur proximité phonologique avec les deux dernières syllabes de legendary. Notons que legendary est tronqué à 11 reprises dans le corpus mais 
n'a été comptabilisé qu'une seule fois puisqu'il s'agit du même procédé qui est redupliqué. Soulignons également que la majorité des cas de troncation relevés (22 occurrences) correspondent à l'étape préalable nécessaire à la formation des amalgames que nous aborderons dans la section suivante.

Précisons par ailleurs que la troncation de brother est présente à 75 reprises dans le corpus. Ces occurrences n'ont toutefois pas été comptabilisées dans les cas de troncation dans la mesure où bro n'est pas un occasionnalisme créé pour les besoins de la série puisque la version tronquée de brother est répertoriée en langue. Nous reviendrons toutefois sur l'utilisation de bro en 4.4. et de legendary en 4.3. en tant qu'outils thématiques de caractérisation du personnage.

\subsubsection{L'amalgame}

L'amalgame, fréquemment considéré comme l'un des procédés de création lexicale les plus créatifs et les plus ludiques ${ }^{8}$ arrive en cinquième position avec 22 occurrences relevées dans le corpus :

(23) Barney: You wanna make this brunch a drunch. (S9E8)

(24) Barney: She left out little Barney. Barnacle Junior. My Barnana is... Barnito Supreme. (S2E13)

(25) Barney: It's the little engine with wood - the whorient express, the long island tail road...

Ted: Oh I got one: Thomas the spank engine.

Ted, that's a children's book [...].

Barney: Ted, Ted, Ted, I thought of some new great names for the drunk train: slam track, the chatta-nookie chu chu. Thomas the spank engine. (S7E16)

(26) Barney: We're going to have one last awesome night together as bros. It's a broing away party. A special broccasion. A bro choice rally. Brotime at the Apollo. (S2E18).

(27) Barney: Welcome to Bangtober Fest. Have a Bangtober Fest T-shirt. Have a Bangtober Fest T-shirt. I'm single again. (S8E2)

(28) Barney: Rockuppied. (S1E17)

(29) Barney: Are you gonna seize this slapportunity or let it slap through your fingers? (S7E9)

L'exemple (23) comporte deux amalgames : brunch qui est entré en langue (et n'a donc pas été comptabilisé) et drunch formé sur le modèle de brunch à partir de troncations de drink et lunch. En (24), Barney découvre un portrait de lui nu, et l'occasionalisme Barnana qui se compose de la première syllabe de Barney et des deux dernières de banana joue le rôle d'un euphémisme dysphémique, stratégie fréquente dans le discours du personnage, comme on le verra en 4.5. L'occurrence (25) présente un télescopage de whore et orient jouant ici le rôle d'un dysphémisme qui, par son caractère déplacé et incongru, suscite le rire. En (28), on note que broccasion se compose de la troncation de brother qui peut être utilisé seul en langage courant et de occasion, les deux termes partageant le $<0>$ dans la forme amalgamée. Enfin soulignons que les exemples (27), (28) et (29) impliquent la troncation du segment initial du deuxième élément seulement, le premier étant une lexie monosyllabique difficile à tronquer tout en conservant le sémantisme. (27) résulte donc de l'association de bang et October donnant une variante de la fête allemande "Oktoberfest", (28) mélange rock et occupied, tandis que (29) amalgame slap et opportunity. 
Il est fréquemment reconnu que l'amalgame est l'un des procédés les plus complexes de création lexicale créative et ludique comme le souligne Renner [2015 : 119-121] :

Lexical blending can be said to be a technique which is both creative and playful.

[...] Because of the wide variety of attested patterns, blending can be claimed to be

the most complex form of wordplay in word-formation.

ou encore l'un des procédés les plus imprévisibles comme l'indique Bauer ${ }^{9}$ [1983] donnant lieu à des « bizarreries » selon Anshen et Aronoff ${ }^{10}$ [1981]. C'est précisément ce caractère imprévisible et cette impression de bizarrerie qui permettent de véhiculer l'humour via les créations lexicales obtenues par amalgame en vertu des théories du contraste et de l'incongruité, mais aussi par une extension de la théorie de la violation bénigne. Bien que la transgression puisse être également thématique, dans le cas de l'amalgame, elle est avant tout morphologique puisque les amalgames sont obtenus par télescopage de fragments de lexies existantes.

\subsubsection{La conversion}

La conversion constitue le sixième procédé le plus fréquemment représenté dans le corpus avec 15 occurrences. Nous avons fait le choix de distinguer la conversion " classique » des phénomènes de downgrading ou de upgrading qui représentent des cas particuliers de conversion et qui seront abordés en 3.1.6.1. et 3.1.6.2. La conversion que nous appelons «classique » implique le transfert d'une lexie d'une partie du discours à une autre, sans modification morphologique mais avec des modifications sémantiques comme dans les exemples ci-dessous :

(30) Barney: My nose is just overflowing with awesome and I had to get some of it out. (S2E11)

(31) Barney: You just did it again. You just mosbied me. (S4E24)

(32) Barney: Girl, I will shamrock your world. (S9E16)

(33) Robin: You were really convincing.

Barney: Please, I was broing you out. I'm just glad he bought it so quick. Any longer, I'd have had to kiss you. (S8E5)

En (30) l'adjectif awesome devient un nom, en (31) le nom propre Mosby, d'après le personnage de Ted Mosby, devient un verbe. L'occurrence (29) est prononcée dans le cadre d'une fête pour la Saint-Patrick et le nom shamrock est employé comme verbe en substitution de rock en raison de la similitude phonologique. Enfin, en (33), la troncation du nom brother est associée à out pour donner l'occasionalisme verbal bro someone out of a situation, Barney souhaitant signifier à Robin qu'il s'est comporté en bon ami pour mettre un terme à un rendez-vous amoureux dont elle n'arrivait pas à se sortir.

Dans le cadre de la conversion, le passage d'une partie du discours existante, à une autre inattendue, semble s'inscrire dans la théorie de l'incongruité et du contraste et celle de la violation bénigne lorsqu'il s'agit de l'humour. En effet, dans la mesure où la partie du discours originelle n'est pas respectée, on peut postuler une extension de la théorie de la violation bénigne, violation qui ne se situerait pas tant au niveau thématique qu'au niveau morphosyntaxique par le jeu avec la plasticité de l'anglais. Notons toutefois que ces créations ad hoc sont humoristiques grâce au contexte dans lequel elles sont prononcées. En effet, le simple fait d'avoir recours au procédé de conversion, comme aux autres matrices lexicogéniques d'ailleurs, ne saurait être humoristique en soi. Le fait humoristique ne semble donc pas tant résulter du recours à 
un procédé donné qu'à une thématique particulière associée à un décalage par rapport aux attendus, comme nous le verrons en 4 .

\subsubsection{La conversion et plus spécifiquement le phénomène de " downgrading"}

Le corpus présente 7 occurrences d'un type de conversion particulier connu sous le nom de downgrading. Il s'agit d'un phénomène qui transforme un syntagme ou une proposition complète en partie du discours. Par exemple, l'occurrence (34) fait jouer la fonction d'adjectifs aux propositions coordonnées «blew out my knee and it hurts like hell » :

(34) Barney: I just had a very minor blew-out-my-knee-and-it-hurts-likehell incident. (S4E19)

(35) Barney: This is not the natural stuff-that-happened-no-more-than-5minutes-ago museum. (S6E8)

(36) Barney: You should have done it in person. Desperate please-don'tleave-me sex is amazing. (S1E4)

(37) Barney: Let me choose your child's middle name because I have thought of the most awesome name of all time.

Marshall: What's the middle name?

Barney: Wait for it.

Marshall: I'm waiting.

Barney: Wait for it.

Marshall: I said I'm waiting.

Barney: Wait for it.

Marshall: What's the middle name?

Barney: No, the middle name is Wait For It. Let's say his name is, I don't know - Barney. He'd be Barney Wait For It Eriksen. How awesome is that?

Marshall: That is the coolest middle name of all time. (S7E23)

En (35), la proposition complexe pourrait être remplacée par un élément nominal dans le cadre d'un nom composé. Il se fonde sur le composé existant Natural History Museum et crée un contraste inattendu. En (37), la locution Wait for it fréquemment utilisée par Barney en tant qu'incise métadiscursive ou qu'infixe discursif, comme on l'a vu en 3.1.3., devient un nom propre puisqu'il l'utilise comme un prénom. De nouveau, l'humour découle ici du caractère inattendu de ce cas de conversion, le phénomène de downgrading étant moins fréquent que le passage d'une lexie simple d'une partie du discours à une autre. On note d'ailleurs que la surprise et l'inattendu sont d'autant plus forts dans cet extrait que Barney utilise régulièrement cette structure dans un autre contexte, d'où l'incompréhension de Marshall qui s'attend à un emploi typique du discours de Barney et espère donc une suite qui ne vient pas.

\subsubsection{La conversion et plus spécifiquement le phénomène de "upgrading"}

On note par ailleurs un cas de upgrading qui consiste à promouvoir un fragment de lexème au statut de lexie indépendante comme c'est le cas de chacune des syllabes de legendary dans l'occurrence (38), qui sont promues au rang de nom dans ces créations ad hoc:

(38) Barney: Dude, we haven't hit the legendary yet. We're only at the le. We still got the gen, the da, the ry. (S1E18) 
Comme pour les autres cas de conversion, on perçoit bien l'incongruité et la violation de la morphologie permettant l'expression de l'humour.

\subsubsection{La siglaison / les initiales}

(39) Barney: That was BS. Before Stinson. (S4E16)

(40) Barney: Daughters, lock up your MILSWANCAS. Mothers I'd Like to Sleep With and Never Call Again. (S5E8)

Dans les créations lexicales présentes en (39) et en (40), une explication est nécessaire à la compréhension du sens et du message humoristique, pour des raisons différentes. En (39), BS qui est normalement une version abrégée et euphémique de bullshit est réinterprété et renvoie aux initiales du personnage, par analogie avec le modèle $B C$ Before Christ. Le téléspectateur s'attend au dysphémisme bullshit, avant de découvrir la réinterprétation surprenante des initiales. En (40), le téléspectateur a besoin d'une explicitation présente dans le cotexte droit pour comprendre le sigle, et c'est la thématique sexuelle qui permet de créer un décalage car elle est déplacée. Dans les deux cas, l'humour ne découle pas de la siglaison ou des initiales en tant que telles mais davantage des explicitations qui s'insèrent dans les théories de l'incongruité en raison de leur dimension inattendue et de la violation bénigne par le recours au non politiquement correct.

\subsubsection{La suffixation}

Six occurrences de création lexicale par suffixation ont été relevées dans le corpus :

(41) Barney: I should feel tremors of psychitude rock my body like a seizure. That was like I declawed a pregnant cat on a porch swing idly swatting at a fly on a lazy Sunday afternoon. (S1E17)

(42) Barney: The fortress of Barnitude? No way. (S2E5)

(43) Barney: You were wonderful. Well you don't get to be a state certified orgasmologist without learning how to please a woman. (S8E19)

En (41) et (42), nous sommes face à deux cas de dérivation par suffixation à l'aide du suffixe -itude issu du latin et utilisé en langage populaire pour créer des noms abstraits. En (43), l'ajout de -ologist à orgasm permet la création d'un nom et par la même occasion d'un nouveau concept qui dénote une science inventée par Barney. Une nouvelle fois, les théories de l'incongruité et de la violation bénigne s'appliquent parce que de nouveaux concepts sont créés découlant d'un jeu avec la structure morphologique des noms ou bien parce qu'ils enfreignent les règles du politiquement correct par le domaine tabou auquel ils appartiennent.

\subsubsection{L'emprunt ou pérégrinisme}

Le personnage de Barney Stinson a également recours à 5 occurrences relevant ou se rapprochant de l'emprunt :

(44) Barney: We are gonna get cirque du solaid, what up! (S3E1)

(45) Barney: Ted Evelyn Mosby. You broke the broath. [...]. Are you aware that breaking a broath can have deadly, even fatal repercussions. Have you 
studied history, Ted?

Ted: Extensively, but I'm a little shaky on fake history so...

Barney: The tragic cost of a broken broath dates back to ancient Broman times. (Flashback)

Roman Barney: Hey Brotus, you'd tell me if like a bunch of dudes were conspiring to assassinate me [...] Just to be sure, can you swear a broath to me? Et tu Brote? (S7E19)

En (44), on note un emprunt au français qui s'accompagne d'un jeu de mots. En effet, l'expression initiale «cirque du soleil » est transformée en «cirque du solaid " par le biais d'un jeu sur la similitude phonologique du français « soleil » et de l'anglais so laid. L'occurrence (45) n'est pas un emprunt à proprement parler étant donné que Brote est un occasionnalisme. On peut considérer qu'il se rapproche d'un pérégrinisme et se fonde sur un emprunt au latin. On note l'emploi adéquat de la déclinaison du vocatif de Brutus. C'est ainsi davantage la syntaxe latine qui est empruntée que le lexique. Cet emprunt s'accompagne de la troncation de brother et de l'amalgame de brother et Brutus. Le cotexte gauche et le renvoi à ancient Broman times, ainsi que la dimension visuelle, permettent de comprendre cette référence au latin, puisque dans cette scène les personnages évoluent dans un décor de la Rome antique et portent des toges. De nouveau, plus que les matrices lexicogéniques elles-mêmes, c'est leur association à un contexte qui se veut humoristique qui provoque le rire par l'incongruité linguistique et thématique créée.

\subsubsection{L'onomatopée}

On relève seulement 2 occurrences d'onomatopée dont une reproduite ci-dessous :

(46) Barney: Courteney Dove, no, I already lost Kurt Coobain. I can't lose you as well. (S9E7)

Lors de son mariage, Barney prévoit un lâcher de colombes et il nomme deux d'entre elles Courteney Dove et Kurt Coobain en référence au couple de chanteurs Courtney Love et Kurt Cobain. L'humour est ici véhiculé par l'incongruité du jeu phonologique qui rapproche le cri de la colombe et le patronyme du chanteur.

\subsubsection{La métonymie}

Pour finir, une seule occurrence de métonymie a été relevée dans le corpus :

(47) Barney: She left out little Barney. Barnacle Junior. My Barnana is... Barnito Supreme. (S2E13)

Dans cette scène, Barney pose nu pour Lily qui a accepté de peindre son portrait, mais elle a volontairement omis de peindre son sexe. Little Barney est alors une forme euphémique de penis, au même titre que les noms suivants et l'humour découle du jeu sur le tabou et du recours à la violation bénigne.

\subsubsection{Autres stratégies de créativité lexicale}

36 À ces matrices lexicogéniques s'ajoutent des occurrences répondant davantage à de la créativité lexicale à partir de lexies existantes qu'à de la création lexicale à proprement parler.

On relève notamment 3 occurrences d'erreurs grammaticales volontaires : 
48) Barney: He's the awesomest most best lookingest greatest guy ever.

(S2E10)

En (48) Barney joue avec la plasticité de la syntaxe de l'anglais et marque le superlatif à 5 reprises, y compris sur des parties du discours n'acceptant normalement pas la forme superlative, et ce, à des fins intensificatrices.

Le corpus comporte également 41 occurrences de jeux de mots fondés sur des similitudes phonologiques à partir de lexies existantes, dont 16 cas de détournement d'expressions, de chansons, de titres d'ouvrages appartenant à la culture populaire ou encore des détournements de noms de marques comme en témoignent les exemples cidessous :

(49) Barney: Challenge accept - wait for it - (pointing to Ted)

Ted: I don't get it.

Barney: Oh Ted. AccepTED. (S4E23)

(50) Barney: No Virginia, you're delightful. I'm delighted. And he's just Ted.

I'm really not planning these things. They just keep happening. (S2E3)

(51) Barney: If you see them bounce, she's about to pounce. (S2E6)

(52) Waitress: Hi Kristen

Krirsten: Everyone pronounces it wrong. The R is after the I.

Waitress: Kirsten.

Krirsten: and before the I. It's Krirsten.

Waitress: Your table's ready.

Barney: Wow, they're trerrible (S8E24)

(53) Barney: I explained. I said, Madeline, every international conflict essentially boils down to sexual tension.

Ted: All international conflicts?

Barney: All, man.

Ted: So, the crisis in the Middle East could be solved by ...

Barney: Gaza Strippers. Next.

Ted: Apartheid?

Barney: Apart thighs. (S4E12)

(54) Barney: Top 10 things I would have called my truck if Ted hadn't been a jerk and gives it back:

10-The Winabango

9-The Pick Up Truck

8-The Ford Explore Her

7-The You Scream Truck

6-Feels on Wheels

5-The Ride Her Truck

4-The 18 Squealer

3-The Esca-laid

2-The Slamboni

1-The 69 Chevvy. (S2E18)

(55) Barney: Here's the mini cherry on top of the regular cherry on top of the sundae of awesomeness that is my life. (S1E15)

(56) Barney: 20 minutes later, the Eagle landed, we knocked space boots, Houston, we have a moaner, other space related double entendres. (S5E19)

(57) Barney: Bro McDonald had a farm, ee i ee i bro, and on that farm he has some chicks, ee $\mathrm{i}$ ee $\mathrm{i}$ bro, with a hot chick here and a dumb chick there. (S8E4)

(58) Barney: Bro, bro, bro your boat, gently to the bar, hit on sluts then do 10 shots. (S8E4) 
(59) Barney: So I figured what is the quickest way to get around the world in a 180 lays. (S5E24)

Les occurrences (49) à (59) présentent des jeux phonologiques avec notamment la prononciation du-ED assimilée à celles de Ted /ted/ en (49) et (50) ou l'ajout d'un <r> à terrible pour faire écho à Krirsten donnant ainsi lieu à la création ad hoc de trerrible. L'exemple (53) se révèle particulièrement intéressant du point de vue de sa construction. Barney explique à Ted que tous les conflits internationaux peuvent être réduits à une tension sexuelle. Il procède à deux jeux de mots sur la phonologie de Apartheid qui devient Apart thighs ainsi que Gaza strippers qui fait écho à la Bande de Gaza (Gaza Strip) en jouant sur l'homophonie et la polysémie de strip. Il semble qu'il s'agisse ici non pas d'une syllepse grammaticale, mais davantage d'une syllepse que nous qualifierons de structurelle avant d'être d'une syllepse oratoire en ce que deux structures différentes coexistent et peuvent donner lieu à des interprétations sémantiques différentes simultanément, ce qui est le propre de la syllepse oratoire. En effet, deux analyses semblent possibles pour Gaza Strippers. On peut soit envisager un procédé de suffixation sur le composé Gaza strip afin de désigner les habitants, ou bien un procédé de composition référant aux strip-teaseuses de Gaza. On est donc en présence de deux procédés de construction lexicale qui résultent de processus cognitifs différents mais qui partagent une morphologie commune en surface. C'est cette syllepse structurelle qui permet de convoquer les deux images mentales simultanées et qui provoque ainsi la syllepse oratoire. Il semblerait alors que cet exemple exhibe un cas de double syllepse, d'abord structurelle puis oratoire.

L'extrait (55) contient plusieurs détournements: the Winnebango qui découle de la marque de camping-car Winnebago, the Ride Her Truck issu de Ryder Truck, The Ford Explore Her qui s'inspire du modèle de voiture Ford Explorer, ou encore the You Scream Truck qui est une réécriture de Ice Cream Truck. Les exemples (57) et (58) font, quant à eux, directement référence aux chansons populaires old MacDonald Had a Farm et Row Row Row Your Boat en jouant notamment avec la sonorité de bro auquel Barney a fréquemment recours. Enfin, (59) détourne le titre du roman de Jules Verne Around the World in Eighty Days par le biais du jeu sur la phonologie de lays et days. Tous ces exemples s'inscrivent dans la thématique des dysphémismes sexuels ou sexistes, thématique récurrente dans le discours du personnage de Barney comme on le verra en 4.5 .

\subsection{Bilan intermédiaire de l'analyse des procédés de création lexicale}

Cette étude a montré que les diverses matrices lexicogéniques sont utilisées à des fréquences différentes dans le corpus pour véhiculer l'humour. L'amalgame étant souvent considéré comme l'un des procédés les plus créatifs, nous nous attendions à ce qu'il soit quantitativement prépondérant. Or, l'amalgame n'arrive qu'en cinquième position représentant $6,2 \%$ des 354 occurrences du corpus, derrière la métaphore $(21,7 \%)$, la composition $(19,7 \%)$, l'infixation $(7,9 \%)$ et la troncation $(7,6 \%)$.

L'analyse des différentes occurrences a également permis de montrer que les mécanismes de création de l'humour sont à rattacher aux théories du contraste, de l'incongruité et de la violation bénigne d'un point de vue morphologique, mais également thématique. 

$\mathrm{du}$ discours de Barney Stinson ne reposent pas uniquement sur des procédés de création lexicale. En effet, aucune création lexicale ne saurait véhiculer l'humour à elle seule. Afin de pouvoir être qualifiées d'humoristiques, elles doivent être employées dans un contexte qui se prête à l'humour et s'ancrer dans des thématiques propices à susciter le rire.

41 Si la combinaison des divers procédés de création lexicale à un contexte propice au rire peut être suffisante à l'émergence de l'humour, ce seul critère ne semble toutefois pas satisfaisant pour caractériser un personnage de fiction. En effet, la caractérisation ne saurait s'effectuer par le biais exclusif du recours aux matrices lexicogéniques dans un contexte défini. Au contraire, ces matrices sont à envisager davantage comme des outils linguistiques qui sont les vecteurs de thématiques particulières permettant à leur tour de caractériser le personnage mais ne peuvent se suffire à elles-mêmes. En ce sens, la caractérisation du personnage de Barney par les auteurs de la série ne semble pouvoir s'effectuer dans l'écriture que par la récurrence de certaines thématiques et stratégies de création lexicale qui deviennent des particularités idiolectales de Barney Stinson.

\section{Particularités idiolectales et caractérisation du personnage}

Afin de comprendre les processus mis en œuvre lors de la caractérisation du personnage, nous prendrons appui sur les travaux de Bednarek [2018:10] selon lesquels les stratégies de construction d'un personnage de fiction se démultiplient sur plusieurs niveaux :

How are TV characters built? Mittell (2015: 130-1) suggests that audience members infer characters' interior states through explicit exterior marks (dialogue, actions, appearance), the dramatic context, and their knowledge of characters. This is more or less in line with cognitive stylistic models of characterisation (Culpeper 2001). While dialogue 'cues' (Culpeper 2001:35) are thus not the only means of constructing character, they are one way in which characters are built. Such cues function to indicate character directly/explicitly (naming the trait) or indirectly/ implicitly (displaying the trait) (Culpeper 2001:163-229), and may be reiterated throughout the TV narrative (Thompson 2003: 27). [...] A wide range of linguistic features can contribute to characterisation in drama, including conversational structure, affective language, lexical richness/diversity, terms of address, syntactic structure, accent/dialect, impoliteness strategies, and (non-)adherence to conversational maxims.

La notion de récurrence, les traits linguistiques ainsi que la notion d'impolitesse soulignés en fin de citation seront particulièrement utiles pour l'analyse des données de ce corpus. Parmi les thématiques privilégiées dans le discours de Barney Stinson, les divers exemples étudiés ont montré une récurrence des termes suits, legendary, bro, ou des structures idiolectales construites sur le modèle de high five, wait for it, ou encore suit up, ainsi qu'une forte prédominance de dysphémismes ou d'euphémismes dysphémiques majoritairement sexuels et/ou sexistes. Ce sont ces thématiques et ces structures idiolectales que nous nous proposons d'étudier dans cette section. 


\subsection{Expressions idiolectales formées sur le patron V + UP (Suit up)} sur la stratégie de détournement afin de véhiculer l'humour.

Parmi les 70 occurrences de composition présentes dans le corpus, 19 sont construites sur le modèle de suit up et en constituent des variations qui diffèrent selon les contextes comme l'attestent les exemples ci-dessous :

(60) Barney: We're building an igloo in Central Park. It's gonna be legendary. Snow suit up! (S1E3)

(61) Barney: You're going as my wingman, flight suit up! (S1E6)

(62) Barney: I penguin-suited up. (S1E6)

(63) Barney: I'm birthday suiting up. (S1E14)

(64) Barney: Now, ladies, slut up! (S1E19)

(65) Barney: Space-suit up Ted, cos you're going to the moon! (S3E3)

(66) Barney: Patch up! (S3E4)

(67) Barney: Lawsuit up! (S3E8)

(68) Barney: Saucy little minx. You sundressed up (S6E1)

Le personnage de Barney prend modèle sur un paradigme existant pour générer des créations lexicales. On note ici le défigement d'une expression en langue par la modification de l'un des termes la composant, tout en conservant le schéma morphologique ( $X$ suit up / $X$ up) afin que celui-ci soit reconnaissable pour le récepteur du message par analogie (voir Sablayrolles [2018], ou encore Zhu [2011 ; 2016]).

Cette structure représente une particularité idiolectale du personnage et permet sa caractérisation. En effet, dès les premiers épisodes et tout au long de la série, Barney apparaît comme un personnage qui revendique l'élégance vestimentaire, toujours vêtu de costumes et de cravates, allant jusqu'à leur vouer un culte et même à leur donner des noms.

Dès le premier épisode, les auteurs posent les bases des traits de personnalité de Barney à la fois visuellement et linguistiquement comme en témoigne l'exemple (69), l'expression suit up étant mentionnée pas moins de trois fois en 20 minutes :

(69) Barney: Just once, when I say 'suit up' I wish you'd put on a suit. (S1E1)

(70) Quinn: I already know you.

Barney: Oh really?

Quinn: You wear a suit all the time.

Barney: Obviously [...].

Quinn: Telling yourself your life is somehow epic or classic or legendary.

Barney: I never say epic or classic. Do you practice the dark arts? (S7E16)

(71) Barney's brother: Once there was this boy who wore a lot of suits and said stuff was legendary. He gave lots of high fives and swore that he would never get married. He really thought he meant it. (S9E12)

En (69), Barney est vêtu d'un costume et souhaiterait que Ted fasse de même. L'emploi de just once et du présent simple dans when I say suit up, associé à la mention des costumes laisse entendre qu'il s'agit d'une des propriétés définitoires du personnage en raison de la récurrence de ses propos. Ceci est confirmé par les occurrences (70) (you wear a suit all the time / obviously), (71) dans l'extrait chanté par le frère de Barney avant son mariage (Once there was this boy who wore a lot of suits). 


\subsection{Expressions idiolectales formées sur le patron $X$ five ou High $X$ (High five)}

Les expressions idiolectales fondées sur le patron de suit up ne sont pas les seules à émailler le discours de Barney Stinson. L'étude du corpus a révélé la récurrence d'un défigement de la structure high five (28 occurrences) comme l'illustrent les exemples suivants :

(72) Barney: Dude, you are so in. That is so awesome. A high five doesn't even cut it. High six. (S6E2)

(73) Lily: High five.

Barney: Sorry, I only give high twos. (S1E15)

(74) Barney: It's gonna be legendary. Phone five - you didn't phone five, did you? I know when you don't phone five, Ted. (S1E3)

(75) Barney: So Robin, you ever report on train wrecks cos I just saw one. What up? Tiny five.

(76) Barney: Solemn low five. (S2E14)

(77) Barney: Relapse five. That's where we high five then it's awkward for a little bit, then we high five again. (S3E9)

(78) Barney: It appears to be some sort of Asian looker. Yes, because on not a father's day, you get a thai you'd actually wear. Word play five. (S4E7)

(79) Barney: That proves I will be this awesome when I'm 80. Arthritis five! (S4E4)

(80) Barney: Hey Ted. Door five. Were you there? (S5E1)

(81) Barney: The Big Bro in the sky had answered my prayers. Almighty five.

(S8E2)

(82) Barney: Did you...? (whispers in Ted's ear)

Ted: Big time.

Barney: Nice, high five. Wait, no, no, retraction five. (S8E13)

(83) Barney: Angry self-five. (S8E19)

(84) Barney: For the next 200 years every male member of the Stinson family was afflicted by the Gipsy's curse. An unquenchable curse thirst for booty that no committed relationship could ever satisfy. That is until my brother James met his husband Tom. The day they got married they lifted the curse forever freeing me from the shackles of having sex with lots of different women. Although, sometimes Robin and I still use the shackles. Bondage five. (S9E2)

(85) Barney: Reverend, more like never end. Prayer-five. (S9E6)

Les occurrences (72) et (73) conservent la morphologie du patron high five avec le recours au modèle High $X$. En (72), Barney défige l'expression pour obtenir high twos puisqu'il porte des moufles donnant ainsi l'impression qu'il n'a que deux doigts. Les exemples (74) à (85) conservent également la structure morphologique mais adoptent le patron $X$ five. Notons que ces occasionnalismes ne peuvent être compris que s'ils s'accompagnent d'un contexte suffisant, ce qui ne semble pas être le cas en (77) puisque Barney accompagne Relapse five d'une définition permettant aux autres personnages et surtout aux téléspectateurs de comprendre le sens véhiculé.

Par leur récurrence dans le corpus, ces occasionnalismes participent à l'attribution de propriétés caractéristiques au personnage de Barney comme le soulignent les extraits suivants : 
(86) Barney: Interesting. Everyone has annoying habits but me. Got it. Ok.

Robin: You sometimes talk in a weird high pitched voice.

Lily: And you're constantly using lame catchphrases. (S3E8)

(87) Barney's brother: Once there was this boy who wore a lot of suits and said stuff was legendary. He gave lots of high fives and swore that he would never get married. He really thought he meant it. (S9E12)

Dans les exemples (86) et (87), l'utilisation des temps et des aspects permettent d'attribuer des traits de personnalité à Barney Stinson.

\subsection{Wait for it et legendary}

Le discours de Barney Stinson se compose également de fréquents emplois de wait for it (30 occurrences) souvent couplés à legendary (17 occurrences) en ayant recours, comme on l'a vu en début d'étude, à des procédés de troncation et d'infixation :

(88) Barney: It's gonna be legen - wait 20 years for it dary - legendary. (S8E20)

(89) Barney: I'm - wait for it - in - wait for it - love - wait for it - with wait for it - a - wait for it - certain - wait for it...

Marshall: I know that you're in love with Robin. Lily told me. (S4E20)

(90) Barney: It was legen - wait for it - zof the fall, legends of the fall. (S5E7)

(91) Barney: My average night is like an average guy's bachelor party. So no offense, but if you two planned it, it's gonna ordi - wait for it - nary, ordinary. (S8E22)

51 Ces expressions idiolectales font partie intégrante du discours de Barney et le caractérisent aux yeux des autres personnages comme on peut le constater dans les exemples (92) à (95) :

(92) Quinn: I already know you.

Barney: Oh really?

Quinn: You wear a suit all the time.

Barney: Obviously [...].

Quinn: Telling yourself your life is somehow epic or classic or legendary.

Barney: I never say epic or classic. Do you practice the dark arts? (S7E16)

(93) Babysitter: No, I'm not taking any nanny job.

Lily: I don't understand.

Babysitter: The most amazing thing happened at my next interview after you guys. I fell in love with a single dad who turns out to be a billionaire. Tomorrow we're heading off to Paris in his private jet. It's gonna be as he would say legen - wait for it. (S8E2)

(94) Barney's brother: Once there was this boy who wore a lot of suits and said stuff was legendary. He gave lots of high fives and swore that he would never get married. He really thought he meant it. (S9E12)

(95) Ralph Macchio: Sorry bro, I'm staying. If this is anything like my bachelor party, strippers, booze, definitely no hand stuff, wink, wink, it's gonna be in - wait for it - credible, incredible.

Ted: You guys are actually a lot alike.

Barney: You take that back sir. I am nothing like Ralph Macchio. (S8E22)

(96) Quinn: Is that the Karate kid? He's a lot like Barney, huh?

Barney: You take that back, Madam. I am nothing like Ralph Macchio. (S8E22)

(97) Ralph Macchio: Man, my bachelor party was - wait for it - and I hope you're hungry cos the second part of this word is edible - incredible. 
Self-shake.

(Clown points at similarity)

Barney: You take that back Clown, I am nothing like Ralph Macchio. (S8E22)

(98) Barney: Our wedding is gonna be legendary.

Robin: No wait for it?

Barney: I've got you. I don't need to wait for it anymore. (S9E1)

En (92), Quinn, une femme rencontrée dans un bar dit à Barney qu'elle sait quel genre d'homme il est (I already know you / Telling yourself your life is somehow epic or classic or legendary). La réponse de Barney (I never say epic or classic) laisse entendre que legendary est l'une de ses particularités idiolectales par le biais de la négation des deux autres adjectifs. L'énoncé au présent simple à la forme négative permet l'attribution implicite des propriétés caractéristiques contenues dans telling yourself your life is legendary. L'exemple (93) permet aussi l'attribution de traits langagiers caractéristiques par le biais du would. Les exemples (95) à (97) mettent en scène Ralph Macchio, l'acteur qui a joué le Karate Kid que Robin a invité pour surprendre Barney lors de son enterrement de vie de garçon. Dès sa première intervention, il apparaît comme le double de Barney, ce que ce dernier n'accepte pas car il ne l'apprécie pas (I am nothing like Ralph Macchio) alors que tous ses amis tendent à souligner les similitudes entre eux (You guys are actually a lot alike/ He's a lot like Barney, huh?). Ralph Macchio porte un costume, et exhibe les mêmes particularités linguistiques idiolectales, à quelques différences près. En effet, il utilise l'infixe wait for it, mais avec l'adjectif incredible au lieu de legendary. Il a également recours au nom bro ainsi qu'à la structure self shake ou platonic shake proche des high five que Barney décline tout au long de la série. Au fil des saisons, le personnage évolue et adopte un style de vie plus rangé. Cette évolution est perceptible en (98), puisque Barney utilise l'adjectif legendary pour décrire son futur mariage avec Robin, et celle-ci, habituée à ses expressions idiolectales, s'étonne de l'absence de l'incise métadiscursive wait for it.

\subsection{Bro}

Une autre thématique particulièrement récurrente dans le discours du personnage de Barney Stinson est celle de ses compères masculins auxquels il renvoie par le biais de bro et qui donne lieu à 26 occurrences de créations lexicales dont voici quelques exemples :

(99) Barney: That's the bro code. A bro doesn't tell a mutual bro that a third bro has a crush on him. Just like the third bro doesn't tell the mutual bro that the original bro went bare pickle in front of her. It's quid pro bro. (S1E14)

(100) Barney: Think of me as Yoda. Only instead of being little and green, I wear suits and I'm awesome. I'm your bro. I'm broda. (S3E5)

(101) Barney: Guys, I've got a new bro. A bro that puts all other bros to shame. The bitches love him he buries bones all day. No one chases tail like him. Why aren't you guys laughing? Oh, I probably should have led with this: he's a dog. I've named him Brover. I'll go fetch him. (S8E5)

(102) Barney: I knew it. I could feel it. It's almost like we were hooking up with her together. You felt that right.

Ted: Couldn't have performed if I did.

Barney: Don't you see Ted? We had a bro-nection. (S8E13) 
(103) Barney: A bachelor's party is a man's bro-mitzvah. We can wear bromulkes, spin the bro-del and consult the wisdom of the bro-rah written in hebro. (S8E22)

(104) Barney: It's in here somewhere. It has to be. Damn it. I knew I should have done these in alphabrotical order.

Ted: Who cares about the bro code? It's just some stupid book you made up.

Barney: How dare you? The bro code has been around for centuries. Nay, whatever's more than centuries.

Ted: Please don't launch into a fake history lesson...

Barney: The bro code can trace its lineage all the way back to Broses himself. Article I - Bros before ho's.

Ted: I see what you're doing. You can't prove that I broke the bro code so you're just making up stories about...

Barney: This sacred text was eventually brought to the new world in 1776 by none other than Christopher Brolumbus. (S9E4)

(105) Ted: I love Barney but I'm not going to jail for him.

Barney: What? Really? Why not? I'd go to jail for you.

Ted: No, you wouldn't.

Barney: Yes, I would. Going to jail for your best friend, that's the dream.

Ted: Really? That's the dream?

Barney: Yeah, it's THE most noble, nay broble thing a bro can do for a fellow bro. (S9E13)

(106) Barney: The Elixir was such a success it was awarded the Brobel prize.

True story. (S9E18)

53 caractéristique de son personnage pourtant fictif que les auteurs de la série ont publié pas moins de quatre ouvrages disponibles à la vente en ligne intitulés respectivement The Bro Code, The Playbook: Suit up. Score Chicks. Be Awesome, Bro Code for Parents: What to Expect When You're Awesome, et Bro on the Go. Outre le fait que les titres reprennent les particularités idiolectales du personnage, les auteurs de la série ont poussé la caractérisation à l'extrême, donnant presque vie à ce personnage fictif, puisque le nom de l'auteur affiché en couverture de ces quatre ouvrages est « Barney Stinson ».

\subsection{Les dysphémismes et euphémismes dysphémiques}

Nous avons évoqué les structures langagières récurrentes dans le discours de Barney Stinson, mais sa caractérisation passe également par la présence de thématiques récurrentes véhiculées par ces procédés de création lexicale. Outre la thématique de l'ami masculin, on note une forte prédominance de la thématique sexuelle ou sexiste dans son discours. Rappelons que le procédé de création lexicale prédominant dans ce corpus est la métaphore. Les métaphores qui sont utilisées, sont souvent accompagnées de syllepses oratoires comme on l'a vu en 3.1.1. et permettent une interprétation littérale et une interprétation dysphémique simultanée à visée humoristique (voir Allan \& Burridge [2006]). Elles sont en outre majoritairement sexuelles ou sexistes. Mais la thématique de la sexualité n'est pas uniquement exprimée par des métaphores et peut être abordée par le biais d'autres procédés de création lexicale qui ont été passés en revue en 3. On dénombre au total 93 occurrences de dysphémismes ou d'euphémismes dysphémiques, dont 83 relèvent du domaine sexuel ${ }^{11}$ :

(107) Barney: You know what else? My younger sister just got married and

I'm about to turn 30 sex. Fantastic. (S1E04)

Lexis, $17 \mid 2021$ 
(108) Robin: What does VIP stand for in your little universe?

Barney: I know the P stands for penis. (S1E5)

(109) Barney: What will be sticking to that yacht? The Barnacle. (S1E6)

(110) Barney: Top 10 things I would have called my truck if Ted hadn't been a jerk and gives it back:

10-The Winabango

9-The Pick Up Truck

8-The Ford Explore Her

7-The You Scream Truck

6-Feels on Wheels

5-The Ride Her Truck

4-The 18 Squealer

3-The Esca-laid

2-The Slamboni

1-The 69 chevvy. (S2E18)

(111) Barney: Let's meet your new doppelganger. Or should I say 'doppelbanger'? (S3E6)

(112) Barney: There's a citizenship test tomorrow. You have to do it. I'll help you study. We're gonna stay up all night long. I'm gonna drill you and then we're gonna study. No, seriously, we're gonna do some cramming and then we're gonna study. No, seriously, we're gonna bone up on...

Robin: Ok Barney.

Barney: Sorry, it's a rich area. (S5E5)

(113) Barney: I'm planting my flag in her if you know what I mean. (S5E5)

(114) Barney: Florence Nightinbed with a lucky patient. (S6E7)

(115) Barney: Tell her. Fine, I'll fill her in. And I am so angry I'm not even going to make a joke about filling her in which I did 3 times last night self five! (S7E19)

(116) Barney: I been going to the lusty leopard for 7 years.

Lily: wow, that's like 49 in perv years.

Barney: 69! Self five! (S8E6)

(117) Barney: Those are "plough me heels". (S8E13)

(118) Barney: For the next 200 years every male member of the Stinson family was afflicted by the Gipsy's curse. An unquenchable thirst for booty that no committed relationship could ever satisfy. That is until my brother James met his husband Tom. The day they got married they lifted the curse forever freeing me from the shackles of having sex with lots of different women. Although, sometimes Robin and I still use the shackles. Bondage five. (S9E2)

(119) Barney: The average male brain can only store a finite number of boob images or bpegs. And your hard drive's filled the capacity with Lily's. They won't go away until you overwrite them. (S2E1)

(120) Barney: You are in the heart of Bachelor county. And as a woman you're an illegal immigrant here. Now you could try to apply for a sex visa but that only lasts 12 hours, 14 if you qualify for multiple entry.

Lily: I say give me multiple high fives.

Barney: Wow, you really are desperate. (S2E5)

(121) Barney: Trust me, by the end of the night, your chad will not be hanging. (S1E6)

(122) Lily: It's as if a cockroach and a mouse, you know...

Barney: Did the horizontal ten-legged interspecies chacha? (S1E7)

Les occurrences (107) à (122) permettent de le dépeindre comme un coureur de jupons qui enfreint les règles du politiquement correct. La plupart de ces exemples relèvent des dysphémismes, mais certains comme (121) et (122) relèvent davantage d'euphémismes dysphémiques. Les références à la sexualité peuvent être explicites 
comme en (107), (108), (110) ou encore (118), mais certains exemples sont moins explicites que d'autres. On pense notamment à l'extrait (109). The Barnacle est un surnom que se donne Barney à plusieurs reprises au cours de la série. Ce surnom provient d'un crustacé : le bernacle. À première vue, la motivation ne paraît pas évidente. Elle devient plus claire lorsque l'on apprend que ce crustacé a la particularité de posséder un organe sexuel extensible pouvant atteindre jusqu'à huit fois la taille du corps du mollusque en faisant la créature à l'organe sexuel le plus long proportionnellement à sa taille.

De nouveau, cette particularité discursive de Barney fait l'objet d'une caractérisation explicite par les autres personnages comme l'indiquent les exemples ci-après :

(123) Lily: It gives us a rare insight into the makings of Barney Stinson, who was a cute little basketball player before he became the biggest pervert in the world. (S6E2)

(124) Ted: Kids, it's no secret your uncle Barney is a bit of a dog. (S8E5)

La remarque de Lily et la mention de the makings of Barney Stinson est une sorte de mise en abyme et évoque en filigrane la construction des traits de personnalité par les scénaristes qui font du personnage ce qu'il est.

\section{Conclusion}

Cette étude a tenté de mettre au jour les rouages complexes de l'expression de l'humour par le biais de procédés de créations lexicales et de créativité linguistique permettant, dans le même temps, de caractériser un personnage de fiction. Elle a montré, d'une part, que les seules stratégies de création lexicale ne sauraient être suffisantes pour véhiculer l'humour. Elles doivent répondre à des critères d'incongruité, de surprise, de contraste, de dépassement de certaines limites sur les plans morphologique, syntaxique, phonologique mais aussi thématique, et ce, dans un contexte propice à susciter le rire. Par ailleurs, si l'association des diverses matrices lexicogéniques et d'un contexte adapté peuvent suffire à l'expression de l'humour, cette complémentarité est insuffisante pour caractériser un personnage de fiction. La dimension répétitive de certaines de ces stratégies ou thématiques semble nécessaire à l'attribution de propriétés caractéristiques et définitoires au personnage de Barney Stinson.

Il apparait alors comme un personnage élégant du point de vue vestimentaire, un coureur de jupons invétéré aux remarques souvent sexistes et sexuelles, ou du moins peu politiquement correctes, très attaché à ses amis masculins (bros), qui a souvent recours à des structures idiolectales récurrentes (high five, wait for it) et qui n'hésite pas à enjoliver la réalité (legendary). Les auteurs rappellent habilement toutes ces caractéristiques dans l'une des dernières interventions du personnage dans l'ultime épisode de la série :

(125) Barney: If it wasn't gonna happen with Robin, then it's just not gonna happen with anyone. I'm never gonna be a guy who meets a girl and from the first time I see her I'm just like 'you are the love of my life. Everything I have, and everything I am is yours forever.'

Girl: Really?

Barney: No, I'm just making a point. Move it along. That's not me. I'm the guy who straightens his tie, says something dirty, high fives himself and 


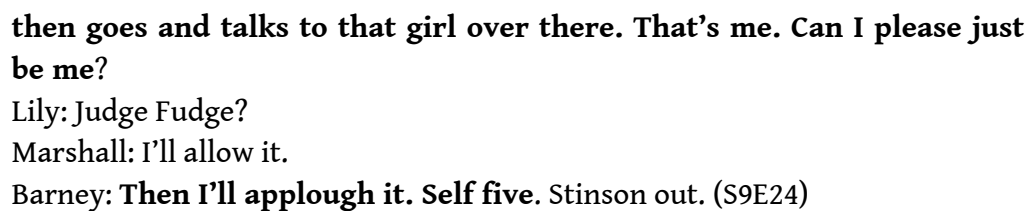

Cet échange dialogué réunit tous ses traits de personnalité et ses particularités idiolectales. Barney souhaite redevenir lui-même et après l'accord de son ami Marshall, il termine sa dernière intervention par un jeu de mots dysphémique et l'expression idiolectale Selffive, qui lui sont propres.

En guise de remarque conclusive, soulignons que si l'humour est véhiculé par l'ensemble des stratégies de création lexicale et autres stratégies de créativité linguistique dans le contexte de ce corpus, les vecteurs d'humour ne se bornent pas à l'utilisation d'outils linguistiques. Les théories de l'incongruité, du contraste et de la violation bénigne s'appliquent à différents niveaux : au niveau linguistique, jouant avec la morphosyntaxe, la phonologie, le sémantisme ; au niveau thématique et discursif par les thèmes récurrents qui enfreignent les règles $\mathrm{du}$ politiquement correct; et également au niveau de la construction et de la caractérisation même du personnage, présenté visuellement comme quelqu'un qui revendique l'élégance physique par son apparence, mais qui dans le même temps tient des propos déplacés, voire choquants, créant ainsi un décalage, une incongruité. Ce personnage au physique élégant se révèle linguistiquement et moralement décalé, incongru, voire inapproprié. Et c'est la synergie de l'ensemble de ces mécanismes qui s'enchevêtrent qui permet l'expression de l'humour et la caractérisation de ce personnage de fiction à l'aide d'outils linguistiques variés.

\section{BIBLIOGRAPHIE}

\section{Corpus}

How I Met your Mother: The Complete Series, 2005-2014, created by Craig Thomas and Carter Bays, CBS

\section{Références}

ALLAN Keith \& BURRIDGE Kate, 2006, Forbidden Words: Taboo and the Censoring of Language, Cambridge: Cambridge University Press.

ANSHEN Frank \& ARONOFF Mark, 1981, "Morphological Productivity and Phonological Transparency”, Canadian Journal of Linguistics/Revue Canadienne de Linguistique 26, 63-72. BAUER Laurie, 2004, A Glossary of Morphology, Washington D.C.: Georgetown University Press. BAUER Laurie, 1983, English Word-formation, Cambridge: Cambridge University Press. BEDNAREK Monika, 2018, Language and Television Series: a Linguistic Approach to TV Dialogue, Cambridge and New York: Cambridge University Press. 
BERGSON Henri, 2000 (1900), Le rire, Quadrige, Presses Universitaires de France.

CHANPIRA E.I., 1966, “Ob okkazional'nom slove i okkazional'nom slovoobrazovanii” [On the occasional word and occasional word formation], in ZEMSKAJA E.A. \& ŠMELEV D.N. (Eds.), Razvitie slovoobrazovanija sovremennogo russkogo jazyka [The development of word formation in Modern Russian], Moskva: Nauka, 153-166.

DRESSLER Wolfgang U. \& TUMFART Barbara, 2017, "New Corpus-Linguistic Approaches to the Investigation of Poetic Occasionalisms: The Case of Johann Nepomuk Nestroy", Yearbook of the Poznan Linguistic Meeting 3(1), De Gruyter Open, 155-166.

GREGAR Jan, 2013, « Humor of the American Sitcom How I Met Your Mother, its Translation and Acceptance by the Czech Audience », Bachelor thesis. https://www.researchgate.net/ publication/ 303374374_Humor_of_the_american_sitcom_How_I_Met_Your_Mother_its_translation_and_acceptance_by_the_Czech_audience HURLey Matthew M., DenNETT Daniel C. \& ADAMs Reginald B. Jr., 2013, « Phénoménologie de l'humour. Qui rit en dernier est le plus lent d'esprit », Terrain, $n^{\circ} 61,16-39$.

MCGHEE Paul E., 1979, Humor, its Origin and Development, W. H. Freeman \& Co Ltd.

RAHMI Awliya, 2017, "Joke Strategies in American Situational Comedy How I Met Your Mother", Jurnal Arbitrer, Vol. 4, 38-51.

REISCH George A. (Ed.), 2014, How I Met your Mother and Philosophy: Being and Awesomeness, Chicago: Carus Publishing Company, Vol. 81, "Popular Culture and Philosophy” series. RENNER Vincent, 2015, "Lexical Blending as Wordplay", in zIRKER Angelica \& WINTER-FROEMEL Esme (Eds.), Wordplay and Metalinguistic / Metadiscursive Reflection: Authors, contexts, techniques and metareflection (Vol. 1), Berlin \& Boston: De Gruyter, 119-133.

SABLAYROLLES Jean-François, 2018, «Créativité lexicale en discours liée à l'existence de paradigmes », Signata, 37-50. https://journals.openedition.org/signata/1345

SAMS Jessie, 2016, Word-Formation in HIMYM, in BEERS FäGERSTEN Kristy (Ed.), Watching TV with a Linguist, Syracuse, NY: Syracuse University Press, 161-180.

SHAFFER David Reed \& KIPP Katherine, 2012, Developmental Psychology: Childhood \& Adolescence, Belmont: Cengage Learning Custom Publishing.

SøRENSEN Majken Jul, 2016, Humour in Political Activism: Creative Nonviolent Resistance, New York: Palgrave Macmillan.

suls Jerry M., 1972, “A Two-Stage Model for the Appreciation of Kokes and Cartoons: An Information-Processing Analysis”, in GOLDSTEN Jeffrey H. \& MCGHEE Paul E. (Eds.), The Psychology of Humor: Theoretical Perspectives and Empirical Issues, New York: Academic Press, 81-100. TOURNIER Jean, 2004 (1968), Précis de lexicologie anglaise, Paris : Ellipses.

VANDEUREN Mikhaël \& VANDEUREN Jean-Pierre, 2016, Théorie générale sur le rire et l'humour :

Unification des théories philosophiques du rire et de l'humour par le développement d'une théorie générale sur ces phénomènes, Casual Intellectual Éditions.

VEATCH Thomas C., 1998, “A Theory of Humor”, Humor: International Journal of Humor Research 11(2), 161-215.

ZHU Lichao, 2011, "Création lexicale et créativité textuelle-cas du figement et défigement », Neophilologica 23, 125-135. 
ZHU Lichao, 2016, « Le défigement dans les schémas prédicatifs », Neophilologica 28, 162-175.

\section{NOTES}

1. Les références de chacun des extraits tirés de la série seront notées sous le format (Saison $X$ Episode X, abrégé ici en S7E19).

2. On distingue le rire Duchenne du rire non Duchenne ou rire conversationnel. Le premier est génétiquement déterminé et peut être assimilé à un réflexe en ce qu'il est déclenché par divers stimuli cognitifs extérieurs (chatouilles, humour, surprise, etc.) et est difficile à contrôler. Le second est socialement déterminé et s'apparente à une forme de politesse. Il vise à apaiser les tensions, ponctuer le discours et à mettre les participants à l'acte discursif dans des dispositions positives. Ce rire est le fruit d'une construction et d'un apprentissage social (voir Hurley, Dennett \& Adams [2013]).

3. Pour un traitement plus complet de toutes les formes d'humour dans la série How I Met Your Mother, voir notamment Rahmi [2017], Reisch [2014], Sams [2016] et Gregar [2013].

4. La notion de besoin ponctuel et immédiat peut être discutable en ce que ces créations font partie d'un écrit scénarisé destiné à être joué sans que le téléspectateur ne se rende compte qu'il s'agit d'un script rédigé et construit en amont par des scénaristes. Si l'on considère que les scénaristes ont fait la démarche de création de ce besoin ponctuel et immédiat dans leur écriture, pas tant pour les interactions entre les personnages que pour la réception du message humoristique par les téléspectateurs, la notion de création ad hoc répondant aux critères définis par Bauer [1983] semble s'appliquer à ce corpus.

5. Nous aborderons dans la Section 4.5. les domaines sources et cibles des métaphores employées qui semblent essentiels à la caractérisation du personnage.

6. Nous verrons en 4.5. les raisons qui sous-tendent le choix de ce surnom pour le personnage.

7. La locution wait for it joue le rôle d'un marqueur métadiscursif au sens large, mais nous parlons ici d'« incise métadiscursive " puisque la locution vient s'insérer à l'intérieur d'une lexie pour ajouter une information en passant, comme le ferait une incise.

8. Voir notamment Renner [2015].

9. Bauer [1983] qualifie l'amalgame de « unpredictable».

10. Anshen \& Aronoff [1981] décrivent les créations lexicales obtenues par amalgame comme des « oddities».

11. Soulignons que les discours de Barney présentent bien davantage de dysphémismes, sexuels ou non, mais dans le cadre de cette étude, nous n'avons retenu que ceux qui relèvent de procédés de création lexicale ou de créativité linguistique.

\section{RÉSUMÉS}

Cet article a pour but de mettre au jour de quelles façons les auteurs de la série télévisée américaine How I Met Your Mother parviennent à créer de l'humour en ayant recours à des stratégies de création lexicale et de créativité linguistique. Nous montrons comment s'articulent les différentes techniques linguistiques pour permettre la caractérisation d'un personnage de fiction, et plus particulièrement Barney Stinson, tout en véhiculant l'expression de l'humour. 
This paper aims to show how the authors and scriptwriters of the popular American TV series How I Met Your Mother managed to create humor by resorting to lexical creation and linguistic creativity strategies. My aim is to highlight how the various linguistic techniques at work intertwine to result in the characterization of a fictional character, namely Barney Stinson, while expressing humor.

\section{INDEX}

Keywords : lexical creation, lexical creativity, humor, occasionalisms, fictional tv series, characterization, word-formation processes, How I Met Your Mother

Mots-clés : création lexicale, créativité, humour, occasionalismes, fiction télévisée, caractérisation, matrices lexicogéniques, How I Met Your Mother

\section{AUTEUR}

\section{LUCILE BORDET}

Université de Lyon (Jean Moulin Lyon 3), Centre d'Études Linguistiques - Corpus, Discours et Sociétés (UR CEL)

lucile.bordet@univ-lyon3.fr 